\title{
Development and Validation of a Useful Taxonomy of Patient Portals Based on Characteristics of Patient Engagement
}

\author{
Michael Glöggler ${ }^{1} \quad$ Elske Ammenwerth ${ }^{1}$ \\ ${ }^{1}$ Institute of Medical Informatics, UMIT-Private University for Health \\ Sciences, Medical Informatics and Technology, Hall in Tirol, Austria \\ Methods Inf Med 2021;60:e44-e55. \\ Address for correspondence Michael Glöggler, MSc, MEng, Institute \\ of Medical Informatics, UMIT-Private University for Health Sciences, \\ Medical Informatics and Technology, Eduard-Wallnoefer-Zentrum 1, \\ 6060 Hall in Tirol, Austria (e-mail: michael.gloeggler@edu.umit.at).
}

\section{Abstract}

Keywords

- taxonomy

- classification system

- patient portal

- EHR portal

- online EHR access
Objective Taxonomies are classification systems used to reduce complexity and better understand a domain. The present research aims to develop a useful taxonomy for health information managers to classify and compare patient portals based on characteristics appropriate to promote patient engagement. As a result, the taxonomy should contribute to understanding the differences and similarities of the portals. Further, the taxonomy shall support health information managers to more easily define which general type and functionalities of patient portals they need and to select the most suitable solution offered on the market. Methods We followed the formal taxonomy-building method proposed by Nickerson et al. Based on a literature review, we created a preliminary taxonomy following the conceptional approach of the model. We then evaluated each taxa's appropriateness by analyzing and classifying 17 patient portals offered by software vendors and 11 patient portals offered by health care providers. After each iteration, we examined the achievement of the determined objective and subjective ending conditions.

Results After two conceptional approaches to create our taxonomy, and two empirical approaches to evaluate it, the final taxonomy consists of 20 dimensions and 49 characteristics. To make the taxonomy easy to comprehend, we assigned to the dimensions seven aspects related to patient engagement. These aspects are (1) portal design, (2) management, (3) communication, (4) instruction, (5) self-management, (6) self-determination, and (7) data management. The taxonomy is considered finished and useful after all ending conditions that defined beforehand have been fulfilled. We demonstrated that the taxonomy serves to understand the differences and similarities by comparing patient portals. We call our taxonomy "Taxonomy of Patient Portals based on Characteristics of Patient Engagement (TOPCOP)."

Conclusion We developed the first useful taxonomy for health information managers to classify and compare patient portals. The taxonomy is based on characteristics promoting patient engagement. With 20 dimensions and 49 characteristics, our taxonomy is particularly suitable to discriminate among patient portals and can easily received

December 22, 2020 accepted after revision March 31, 2021 published online July 9,2021
DOI https://doi.org/ $10.1055 / \mathrm{s}-0041-1730284$. ISSN $0026-1270$.

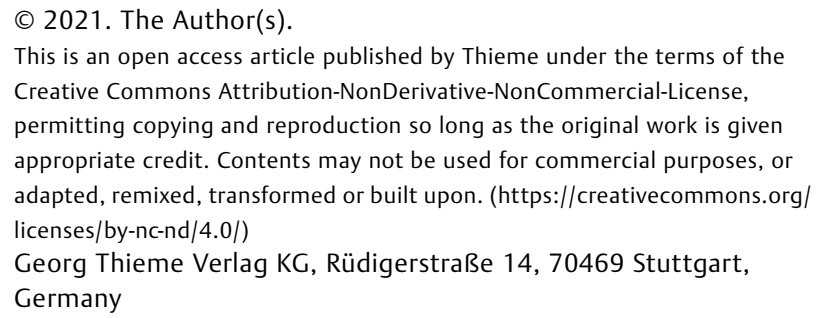


be applied to compare portals. The TOPCOP taxonomy enables health information managers to better understand the differences and similarities of patient portals. Further, the taxonomy may help them to define the type and general functionalities needed. But it also supports them in searching and comparing patient portals offered on the market to select the most suitable solution.

\section{Introduction}

The advent of the internet and the rapid digitization of all areas of life have also lead to significant changes in the health care field worldwide. ${ }^{1}$ The emergence of patient portals in the early $2000 \mathrm{~s}$ ${ }^{2}$ was a logical consequence of the introduction of electronic health records (EHR) in the $1990 \mathrm{~s}^{3}$ A patient portal is an internet-based application allowing patients to have autonomous access to their EHR anywhere at any time. ${ }^{4}$ Besides EHR access, a patient portal may also offer additional functionalities ${ }^{5}$ such as access to test and laboratory result, viewing visit notes, requesting medication refills, appointment scheduling, or secure messaging with the health provider. ${ }^{5-8}$

Patient portals are considered a health information technology that promotes patient engagement by providing tools to become active participants in one's own care. ${ }^{9-11}$ Patient engagement has received considerable attention in recent years, ${ }^{10}$ and patient portals may contribute to engaging the patients actively in their care by granting them access to their health data and providing additional functionalities. ${ }^{7,12-14}$

To date, we find a very heterogeneous landscape and a broad diversity of patient portals ${ }^{15}$ regarding their intended deployment and functionalities. ${ }^{8,16}$ Patient portals can be projected for specific care sectors, for example, primary or secondary care based on each care sector's different needs. They may also follow an approach not specific for a sector. Further, patient portals may also focus on particular diseases and offer disease-specific functionalities to enhance the treatment of specific patient groups, for example, patients with asthma, ${ }^{17}$ renal conditions, ${ }^{16}$ or diabetes. ${ }^{18}$ These different application areas and scopes of patient portals are mirrored in the diversity of functionalities that patient portals may offer, for example, concerning the method of communication between patients and physicians, the possibility to report and grant access to patient-generated health data in the portal, or the possibilities of patients to manage access to their health data. These different application areas and scopes of portals and the resulting large variability in offered functionality highlight the complexity of patient portals' domain and the challenge to match the specific requirements of the clinical setting to patient portals available on the market. This was confirmed by explorative interviews that we performed from 2018 to 2020 with software vendors of patient portals and with health information managers in Germany, Switzerland, and Austria. In these interviews, we found that many health information managers still feel that they have too little knowledge of patient portals. They thus have difficulties understanding the various application areas and scopes of portals, defining their own general requirements, and select- ing the best patient portal for their specific context or problem. Health information managers are involved in selecting, introducing, and managing patient portals for their health institution. A patient portal taxonomy would help them to more easily define which general type and functionalities of patient portal they need and to search and compare patient portals offered on the market to select the best solution. Classifying patient portals with a taxonomy may show them more clearly the similarities and differences of patient portals and support them in their selection decision. The need for a patient portal taxonomy was also stressed in a recently published Cochrane Review on the impact of patient portals. ${ }^{19}$

At present, there exist just a limited number of publications related to patient portal taxonomies. Ammenwerth et $\mathrm{al}^{4}$ developed a taxonomy that aims to distinguish patient portals in a systematic review dealing with their effect on patient empowerment and health-related outcomes. Roehrs et $\mathrm{al}^{1}$ developed a taxonomy that aims to identify open questions related to personal health record (PHR) data types, features, and architecture types. A PHR or personal health record provides patients with web-based access to their health data that is under the control of the patient, ${ }^{1}$ while an EHR typically is under the control of the provider. ${ }^{5,9,14}$ Scheplitz et al $^{20}$ created a framework for patient portal functionalities to record all possible functions to identify specification gaps related to software development. Walker et al developed a framework to evaluate how well health information technology can support patient engagement by applying five engagement scoring levels. ${ }^{10}$

These attempts only provide parts of a potential patient portal taxonomy and are developed for different users and purposes. Since the user determines the intended purpose of a taxonomy and the purpose guides the development by focusing on a specific phenomenon of interest, ${ }^{21,22}$ different users or purposes may lead to different taxonomies. ${ }^{23}$ Further, a useful taxonomy must yield utility for a specific problem domain. ${ }^{24}$ To sum up, the found taxonomies are not suitable to yield utility for classifying and comparing patient portals based on characteristics appropriate to promote patient engagement and to understand the differences and similarities.

A taxonomy is a classification system to classify similar objects of a domain into groups based on distinct characteristics and offers a set of decision rules. ${ }^{23,25-27}$ Various classification structures have been used to construct taxonomies in various domains. ${ }^{28,29}$ For our research, we follow Nickerson et $\mathrm{al}^{23}$ who define a taxonomy $\mathrm{T}$ as a set of $n$ dimensions $\mathrm{D}_{\mathrm{i}}$ $(i=1, \ldots, n)$, with each dimension consisting of $k_{i}\left(k_{i} \geq 2\right)$ mutually exclusive and collectively exhaustive characteristics $\mathrm{C}_{\mathrm{ij}}\left(\mathrm{j}=1, \ldots, \mathrm{k}_{\mathrm{i}}\right)$. 


\section{Objective}

This research aims to develop a useful taxonomy for health information managers to classify and compare patient portals based on characteristics appropriate to promote patient engagement. As a result, the taxonomy should contribute to understanding the various application areas and scopes of portals. Further, it should help them to more easily define which type and general functionalities of patient portal they need. However, the taxonomy is not intended to serve as a standard framework for patient portal functional requirement specification. Classifying patient portals with a taxonomy may more clearly show the similarities and differences of patient portals to the health information managers and support them in their selection decision of portals offered on the market.

\section{Methods}

Taxonomies can be developed very informally and ad hoc, based on the specific knowledge in a given domain, or they can be constructed in a highly formal and standardized manner. ${ }^{28}$ For our research, we adopted a more formal taxonomy-building method proposed by Nickerson et al. ${ }^{23}$ This method is particularly suitable for our purpose as it specifies the necessary steps and integrates two optional, iterative development approaches to conceptionally build and empirically evaluate our taxonomy. ${ }^{30}$ Further, the method defines ending conditions determining when our taxonomy is useful and when to end the iterative development cycles. We will now outline the two research cycles and the major iterations we conducted, the ending conditions we defined, and the definition of "usefulness" of the taxonomy. In -Supplementary Appendix A (available in the online version), we describe the method in more detail.

\section{First Research Cycle-Developing the Taxonomy}

Following the method, we first defined the users and the intended use of the taxonomy. Second, we specified the metacharacteristic of the taxonomy, and third, we determined the ending conditions. Then we developed a preliminary taxonomy using two iterations, applying the conceptional-to-empirical approach in each. After each iteration, we checked if taxonomy revision was needed and verified the fulfillment of all ending conditions. We now describe these steps in more detail:

\section{Defining Users and Intended Use}

The method by Nickerson et al requires a clear definition of the user and the intended use of the taxonomy. ${ }^{23}$ Our taxonomy's projected users are health information managers. They are responsible for obtaining, monitoring, and securing the clinical databases and the EHRs to ensure that health care providers and patients can access patient health information when and where they need it. $^{31}$ Their intended use is to classify and compare patient portals based on the characteristics and functionalities of the portals that seem appropriate to support patient engagement. This allows the user to compare patient portals (e.g., during system selection) and better understand the differences and similarities.

\section{Defining Meta-characteristic}

The taxonomy's intended use is the central determinant for choosing the meta-characteristic, because all dimensions and characteristics must be a logical consequence of the meta-characteristic. $^{23,32}$ We thus defined the following meta-characteristic: portal characteristics useful to compare patient portals based on their ability to promote patient engagement.

\section{Defining Ending Conditions}

Since the method is iterative, we specified ending conditions, presented in -Tables 1 and 2, to determine when to end the iterative development process and when the taxonomy is considered a "useful taxonomy." Our objective was not to create the best taxonomy, but a "useful taxonomy." Since there is no objective metric to determine the usefulness or quality of a taxonomy, ${ }^{26}$ we adopted the subjective ending conditions ( - Table 2) for a "useful taxonomy" as proposed by Nickerson et al. ${ }^{23}$

\section{First Iteration of Taxonomy Development}

In the first iteration, we created an initial taxonomy based on the patient portal taxonomy proposed by Ammenwerth et al. ${ }^{4}$ We selected suitable dimensions applying the conceptional-to-empirical approach. Based on the method by

Table 1 Fundamental and supplementary objective ending conditions for a useful patient portal taxonomy

\begin{tabular}{|c|c|}
\hline $\begin{array}{l}\text { Fundamental objective } \\
\text { ending conditions }\end{array}$ & Description \\
\hline - Collectively exhaustive & All patient portals of interest fall into one of the characteristics within a dimension \\
\hline - Mutually exclusive & No patient portal falls into more than one characteristic within a dimension \\
\hline \multicolumn{2}{|c|}{ Supplementary objective ending conditions } \\
\hline \multicolumn{2}{|c|}{$\begin{array}{l}\text { - All patient portals of interest were examined. } \\
\text { - At least one patient portal is classified under every characteristic of every dimension. } \\
\text { - No new dimension or characteristic was added in the last iteration. } \\
\text { - Every dimension or characteristic was merged or split in the last iteration. } \\
\text { - Every characteristic is unique within its dimension. } \\
\text { - Each taxon is unique and is not repeated. }\end{array}$} \\
\hline
\end{tabular}


Table 2 Subjective ending conditions for a useful patient portal taxonomy

\begin{tabular}{|l|l|}
\hline $\begin{array}{l}\text { Subjective ending } \\
\text { conditions }\end{array}$ & Description \\
\hline It should be concise & $\begin{array}{l}\text { The taxonomy should not contain too many dimensions or characteristics in each dimension } \\
\text { because a taxonomy with too many dimensions and many characteristics would be difficult } \\
\text { to comprehend and to apply. }\end{array}$ \\
\hline It should be robust & $\begin{array}{l}\text { The taxonomy should provide enough dimensions and characteristics to be of interest. } \\
\text { The dimensions and characteristics provide for sufficient discrimination among patient portals. }\end{array}$ \\
\hline $\begin{array}{l}\text { It should be } \\
\text { comprehensive }\end{array}$ & $\begin{array}{l}\text { The taxonomy should include all dimensions and characteristics to classify all } \\
\text { patient portals of interest. }\end{array}$ \\
\hline It should be extendible & $\begin{array}{l}\text { The taxonomy should allow for additional dimensions and new categories within a } \\
\text { dimension when new patient portals appear. }\end{array}$ \\
\hline It should be explanatory & The dimensions and characteristics should explain the objects of interest sufficiently. \\
\hline
\end{tabular}

Nickerson et al, "conceptional-to-empirical” means to create characteristics and dimension by theoretical foundation.

\section{Second Iteration of Taxonomy Development}

The second iteration was based on a qualitative literature review, again applying the conceptional-to-empirical approach. From March 2020 to May 2020, we conducted electronic searches in the databases PubMed (Medline), Cochrane Library, IEEE Xplore, and ACM Digital Library. A preliminary search showed that researchers use different terms to refer to a patient portal. The diversity of terms used made it necessary to extend our search terms to multiple synonyms and acronyms for the search term "patient portal" (-Supplementary Appendix B; available in the online version). We combined in our search the primary search terms with each of the complementary search terms using the Boolean operators (- Supplementary Appendix C; available in the online version).

Our searches identified 40 relevant publications which we analyzed by applying the summarizing content analysis, an inductive analysis method was proposed by Mayring. $^{33-35}$ The meta-characteristic, mentioned above, guided the selection of the characteristics. We identified other characteristics adequate to distinguish among patient portals and created new dimensions. Further, we split dimensions from the first iteration to address the identified characteristics adequately. In - Table 3, we present the results of the literature database search. In -Supplementary Appendix D (available in the online ver- sion), we present the inclusion criteria for publications, and in -Supplementary Appendix E (available in the online version), a list of all publications included in our research and the derived characteristics.

\section{Second Research Cycle-Evaluation of the Taxonomy}

The result of the first research cycle is a preliminary taxonomy that describes the dimensions and characteristics of a patient portal. In the second research cycle, we now evaluated the appropriateness of each dimension and characteristic using two iterations and applying the empirical-to-conceptional approach. Based on the approach by Nickerson et al, "empirical-to-conceptional" means empirically evaluating the theoretically derived characteristics and dimensions through the use of real patient portals. Further, this approach also intends to create new characteristics and dimensions by classifying real patient portals. At the end of the second research cycle, we demonstrate how to use the final taxonomy to compare two patient portals.

\section{Third Iteration of Taxonomy Development}

We started the evaluation by analyzing product descriptions of patient portals offered by software companies and classified the portals with our preliminary taxonomy. From September 2020 to November 2020, we searched the DMEA (digital medical expertise and applications [trade show]) Virtual Market Place for software vendors offering patient portals. DMEA is Europe's most important trade show for digital health care, with 576 exhibitors in $2020 .{ }^{36}$ We present the companies'

Table 3 Results of literature search and publications included in the research

\begin{tabular}{|l|l|l|l|l|l|}
\hline Source & Search order & Results screened & Read title and abstract & Read in full text & Included in research \\
\hline Researchers & 1 & 3 & 3 & 3 & 3 \\
\hline PubMed & 2 & 2,965 & 259 & 90 & 22 \\
\hline Cochrane & 3 & 808 & 72 & 21 & 7 \\
\hline IEEE Xplore & 4 & 552 & 43 & 16 & 4 \\
\hline ACM DL & 5 & 1,276 & 68 & 18 & 4 \\
\hline Total & & 5,604 & 445 & 148 & 40 \\
\hline
\end{tabular}

Abbreviations: ACM DL, Association for Computing Machinery Digital Library; IEEE Xplore, Institute of Electrical and Electronics Engineers Digital Library. 
Table 4 Results of the search for companies and their inclusion in the research

\begin{tabular}{|l|l|l|l|}
\hline Source & $\begin{array}{l}\text { Companies } \\
\text { analyzed }\end{array}$ & $\begin{array}{l}\text { Companies that } \\
\text { offer portals }\end{array}$ & Included \\
\hline Researchers & 4 & 4 & 4 \\
\hline DMEA & 83 & 39 & 13 \\
\hline Total & 87 & 43 & 17 \\
\hline
\end{tabular}

Abbreviation: DMEA, digital medical expertise and applications (trade show).

inclusion criteria in - Supplementary Appendix F (available in the online version).

We explored products and vendors, searching by category and keywords (-Supplementary Appendix G; available in the online version). First, we analyzed the product descriptions published in the Virtual Market Place, following-up on those companies that appear to offer a patient portal. Since the product descriptions published on most companies' web sites were not very clear, we contacted the companies by phone and e-mail to find out if they offer a patient portal. If affirmative, we inquired detailed product information by e-mail. In addition to the search on DEMA, we included in our research companies recommended by other companies or known by the researchers. In -Table 4, we present the results of the search for companies and their inclusion in the research.

We analyzed the product descriptions of 17 patient portals applying Mayring's summarizing content analysis. ${ }^{35}$ Based on the characteristics identified in the portals' product descriptions, we classified the included portals to verify the preliminary taxonomy. Further, we aimed to find other characteristics to create new dimensions and extend existing dimensions.

\section{Fourth Iteration of Taxonomy Development}

For the second evaluation step, we purposefully selected patient portals of health care providers and national health care organizations available online. We applied a convenience sampling strategy and analyzed nine portals that emerged during the literature review and two portals known by the researchers. In - Supplementary Appendix $\mathrm{H}$ (available in the online version), we present the specific inclusion criteria. We classified the included portals based on the characteristics identified in the patient portals' online descriptions to verify existing characteristics and dimensions. Further, we aimed to find new characteristics to enhance the taxonomy, since some of these portals were designed for the organizations' particular needs. To analyze the portals' descriptions, we applied Mayring's summarizing content analysis. ${ }^{35}$

\section{Results}

\section{First Research Cycle-Developing the Taxonomy}

\section{First Iteration}

We built a preliminary taxonomy based on the taxonomy proposed by Ammenwerth et $\mathrm{al}^{4}$ and purposefully selected six from seven dimensions with their characteristics to create our initial taxonomy:
$\mathrm{T}_{1}=\{$ Request (with request options, no request options) Communicate (with communication, no communication) Remind (with reminder options, no reminder options) Educate (with education options, no education options) Share (with data Share options, no data share options) Manage (with management options, no management options)\}

Due to the first iteration's purely conceptual nature, just four from 13 ending conditions were met (-Fig. 1). The method had to be repeated at least one more time.

\section{Second Iteration}

We opted to build upon the existing literature related to patient portals and identified 45 characteristics adequate to follow the determined meta-characteristic logically. We revised the taxonomy and renamed the dimensions, $\mathrm{T}_{1}$ : Communicate to $\mathrm{T}_{2}$ : E-Consult; $\mathrm{T}_{1}$ : Remind to $\mathrm{T}_{2}$ : System Notifications; and $\mathrm{T}_{1}$ : Educate to $\mathrm{T}_{2}$ : Patient Education. We changed the names of the aforementioned dimensions to more precise ones to better reflect the characteristics that the dimensions are built on after the second iteration. Responding to the characteristics that the dimensions were built on, we split the dimensions, $\mathrm{T}_{1}$ : Request into $\mathrm{T}_{2}$ : Appointment Booking and $\mathrm{T}_{2}$ : Prescription Renewal; $\mathrm{T}_{1}$ : Share into $\mathrm{T}_{2}$ : Health Monitoring and $\mathrm{T}_{2}$ : Visit Preparation; and $\mathrm{T}_{1}$ : Manage into $\mathrm{T}_{2}$ : Record Access and $\mathrm{T}_{2}$ : Records Management. Further, we created nine new dimensions. After the end of the second iteration, our preliminary taxonomy $\mathrm{T}_{2}$ was as presented in - Fig. 2. The progress of the dimensions' development is shown in -Fig. 3 .

Since three dimensions were split into six dimensions, and nine new dimensions were created in this iteration, the method must be repeated. Only 4 of 13 ending conditions were met, as assessed in - Fig. 1. Due to the purely conceptional approach of the first two iterations, we needed to examine empirical cases to evaluate the appropriateness of the dimensions and characteristics.

\section{Second Research Cycle-Evaluation of the Taxonomy}

\section{Third Iteration}

To evaluate the taxonomy, we analyzed and classified 17 patient portals offered by software companies ( - Supplementary Appendix I; available in the online version) and identified six new characteristics. To adequately classify the portals, two new dimensions were added. The dimension Patient Target was formed by the characteristics "outpatient" and "in \& outpatient" and the dimension Study Sign-Up by the characteristics "no sign-up" and "with sign up." Since two new dimensions and four new characteristics were created in this iteration, and six ending conditions were not fulfilled ( $~$ Fig. 1), the method was repeated.

\section{Fourth Iteration}

In the next evaluation step, we analyzed 11 patient portals of health care providers (-Supplementary Appendix J; 


\begin{tabular}{ll}
\hline 1 & Objective ending conditions \\
\hline Cundamental objective ending conditions \\
Collectively exhaustive: All patient portals of interest fall into one \\
of the categories within a dimension. \\
Mutually exclusive: No patient portal falls into more than one \\
category within a dimension. \\
\hline Supplementary objective ending conditions \\
All patient portals of interest were examined. \\
Each characteristic was assigned at least to one patient portal. \\
No new dimensions or characteristics was added in the last iteration. \\
No dimensions or characteristics were merged or split in the last iteration. \\
Every dimension is unique. \\
Every characteristic is unique within its dimension. \\
\hline \\
Subjective ending condition \\
Concise: limited number of dimensions, charcteristics in each dimension. \\
among pufficient number of dimensions and charcteristics to discriminate \\
Comprehensive: Identify all relevant dimensions and characteristics of an object. \\
Extendible: new dimensions and categories can be added in future. \\
Explanatory: The dimensions and characteristics sufficiently explain the objects .
\end{tabular}

Fig. 1 Progress of fulfillment of ending conditions by iteration. Reproduced with permission from Roeder et al. ${ }^{29}$

\begin{tabular}{|c|c|c|c|}
\hline DIMENSIONS & \multicolumn{3}{|c|}{ CHARACTERISTICS } \\
\hline Care Sector Target & primary care & secondary care & generic \\
\hline Portal Specialization & \multicolumn{2}{|c|}{ universal } & disease-specific \\
\hline Activity Monitoring & \multicolumn{2}{|c|}{ no insight } & with insight \\
\hline Appointment Booking & no booking & request & schedule \\
\hline Prescription Renewal & no renewal & basic renewal & advanced renewal \\
\hline Portal Customizability & \multicolumn{2}{|c|}{ not customizable } & customizable \\
\hline E-Consult & no e-consult & asynchronous & synchronous \\
\hline System Notifications & no notifications & reminder & alerts \\
\hline Patient Education & no education & non-personalized & personalized \\
\hline Therapy Instructions & \multicolumn{2}{|c|}{ non-protocol-based } & protocol-based \\
\hline Health Monitoring & no monitoring & self-reported & self-tracked \\
\hline Visit Preparation & \multicolumn{2}{|c|}{ no preparation } & with preparation \\
\hline Declaration of Will & \multicolumn{2}{|c|}{ no registration } & with registration \\
\hline Second Opinion & \multicolumn{2}{|c|}{ no inquiry } & with inquiry \\
\hline Record Access & no control & shared control & full control \\
\hline Records Management & \multicolumn{2}{|c|}{ no management } & with management \\
\hline Health Data Amend & review & correct & delete \\
\hline Health Data Up Load & \multicolumn{2}{|c|}{ no upload } & with upload \\
\hline
\end{tabular}

Fig. 2 Preliminary taxonomy $T_{2}$ after the second iteration. 


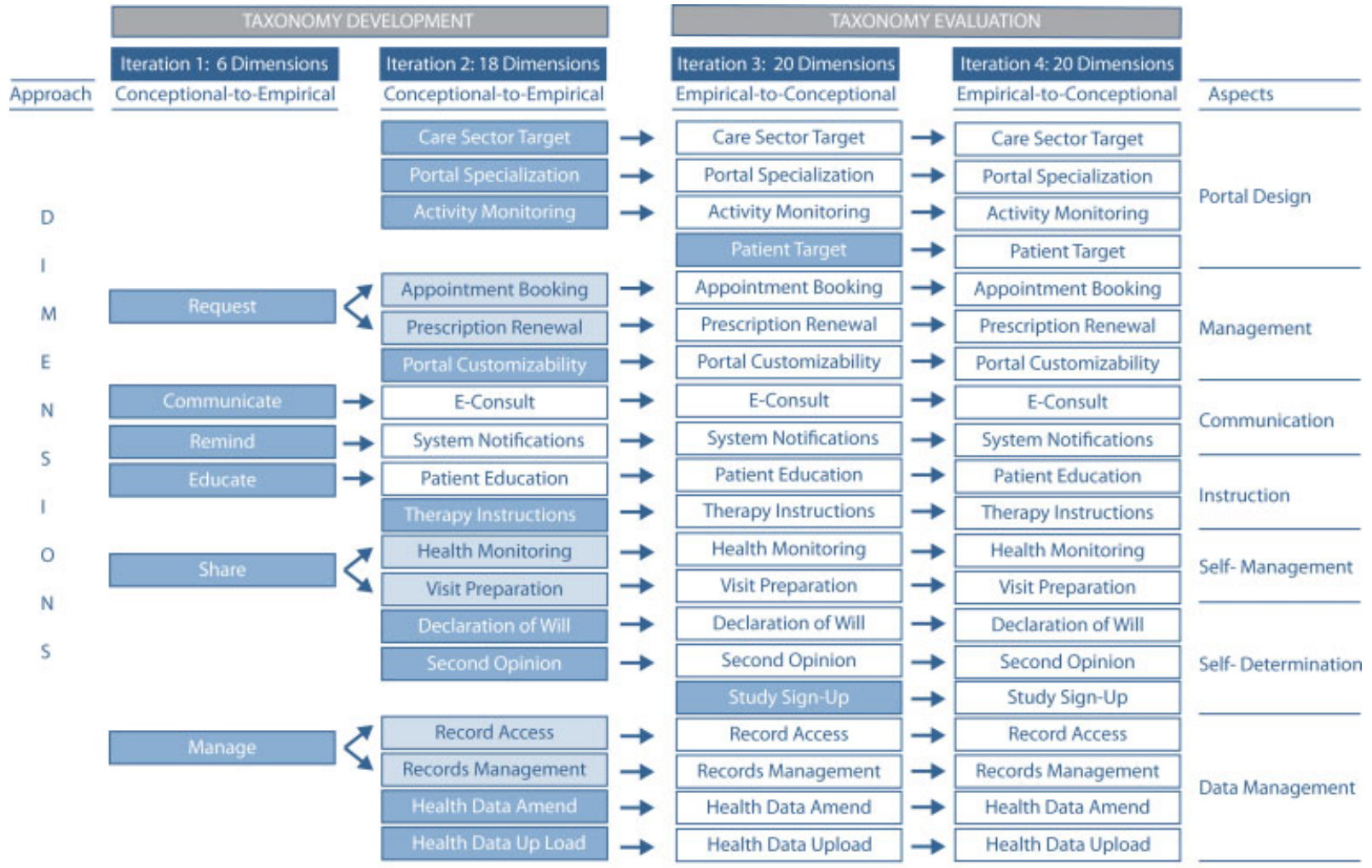

Legend $\square$ new dimension from current iteration $\square$ dimension from previous iteration $\square$ split dimension from previous iteration

Demonstration of the dimensions' progress as proposed by Remane et. al $2016{ }^{30}$

Fig. 3 Progress of the dimensions for the patient portal taxonomy.

available in the online version). No new characteristics were found, and no dimension was changed. The evaluation conducted by iterations three and four was aimed to prove each characteristic's appropriateness by classifying real patient portals. In - Fig. 4 , we assigned the reference IDs of the classified companies and health provider portals to the taxonomy's characteristics to indicate the matching with the characteristics of the examined portals. With this, we proved that real patient portals verified all characteristics. Further, we demonstrated that all patient portals of interest fall into one category within a dimension, and that no portal falls into more than one category within a dimension.

To decide if our taxonomy is finished, we checked all the ending conditions. After examining all patient portals of interest, the taxonomy can be considered collectively exhaustive and mutually exclusive, and at least one patient portal was assigned to each characteristic as demonstrated in -Fig. 4. Further, every dimension and characteristic is unique, and no dimension or characteristic was split, merged, or added in the last iteration. At this point, our taxonomy fulfills all objective ending conditions as defined beforehand.

We further verified the subjective ending conditions to assess if our taxonomy is useful. With 49 characteristics and 20 dimensions, the taxonomy is concise and robust, offering a limited but at the same time sufficient number of dimensions and characteristics to discriminate among patient portals. The total number of dimensions and characteristics per dimension allows future extension if new characteristics come up. The taxonomy is, therefore, expandable. After reviewing the related scientific literature and analyzing all portals of interest, we identified all dimensions and characteristics. Therefore our taxonomy is comprehensive. Finally, the identified dimensions and characteristics sufficiently explain patient portals, as a result of which the taxonomy can be considered explanatory. At this point, the taxonomy $\mathrm{T}_{4}$ satisfies all objective and subjective ending conditions ( - Fig. 1) and can be considered finished and useful. A comprehensive description of all dimensions and characteristics is presented in -Supplementary Appendix $\mathrm{K}$ (available in the online version).

\section{How to Use the Final Taxonomy to Compare Two Patient Portals}

In - Fig. 5, we present our final and useful taxonomy and show how to perform a comparison. To make the taxonomy simple to comprehend and apply, we assigned a numbering to the dimensions and seven aspects of patient engagement. We call our taxonomy "Taxonomy of Patient Portals based on Characteristics of Patient Engagement (TOPCOP)." The comparison of two patient portals can be performed by assigning each portal's characteristics to the taxonomy's characteristics, where they match. This shows where the examined patient portals have matching or different characteristics. In performing the comparison, we demonstrated that our taxonomy is suitable to compare patient portals and that the differences and similarities become apparent. 
The columns "COMPANY | PORTAL" indicate which of the 17 portals that were analyzed based on company product information (\#1-17, in blue) and which of the 11 directly examined patient portals offered by healthcare providers (\#18-28, in orange) match the indicated characteristics of the taxonomy's dimensions.

\begin{tabular}{|c|c|c|c|c|c|c|}
\hline DIMENSION & CHARACTERISTIC & COMPANY | PORTAL & CHARACTEPISTIC & COMPANY | PORTAL & GARACIEPSTC & COMPANY | PORTAL \\
\hline Care Sector Target & primary care & $5,9,13$ & secondary care & $10,14-17,18,21,27$ & generic & $1-4,6-8,11,12,19,20,22-26,28$ \\
\hline Portal Specialization & universal & $2-4,6-12,14-16,18-21,23-28$ & disease-specific & \multicolumn{3}{|l|}{$1,5,13,17,22$} \\
\hline Activity Monitoring & no insight & $1,2,5,7,9-11,13,14,21,22,25,27,28$ & with insight & \multicolumn{3}{|c|}{$3,4,6,8,12,15,16,17,18-20,23,24,26$} \\
\hline Patient Target & outpatient & $5-10,13,14,18-24,26-28$ & in \& outpatient & \multicolumn{3}{|l|}{$1-4,11,12,15,16,17,25$} \\
\hline Appointment Booking & no booking & $14,19,20,26,28$ & request & $2,6,11-13,22,23$ & schedule & $1,3-5,7-10,15-18,21,24,25,27$ \\
\hline Prescription Renewal & no renewal & $1,4,12,14-18-20,22,28$ & basic renewal & $2,5,7,10,11,21,25,27$ & advancedienewal & $3,6,8,9,13,23,24,26$ \\
\hline Portal Customizability & not customizable & $5-9,11,12,14,16,18,19,21-25,27,28$ & customizable & \multicolumn{3}{|l|}{$1-4,10,13,15,17,20,26$} \\
\hline E-Consult & no e-consult & $2,11,14,19,26,28$ & asynchronous & $4,17,22,23$ & synchronous & $1,3,5-10,12,13,15,16,18,20,21,24,25,27$ \\
\hline System Notifications & no notifications & $14,19,28$ & reminder & $1,3-8,11,12,15-18,20-27$ & alerts & $2,9,10,13$ \\
\hline Patient Education & no education & $4,5,7,11,14,15,19,20,23-26,28$ & non-personalized & $3,9,18,21,27$ & personalized & $1,2,6,8,10,12,13,16,17,22$ \\
\hline Therapy Instructions & non-protocolbased & $1,3-7,11-15,17,18-23,25-28$ & protocol-based & \multicolumn{3}{|l|}{$2,8,9,10,16,24$} \\
\hline Health Monitoring & no monitoring & $9,11,12,18-20,23$ & self-reported & $4,6,13,15,22,24,25$ & self-tracked & $1-3,5,7,8,10,14,16,17,21,26-28$ \\
\hline Visit Preparation & no preparation & $11,14,19,21,23,24,26,28$ & with preparation & \multicolumn{3}{|c|}{$1-10,12,13,15-18,20,22,25,27$} \\
\hline Declaration of Will & no registration & $2,3,5-10,12-14,17,18,21,22,25,27$ & with registration & \multicolumn{3}{|c|}{$1,4,11,15,16,19,20,23,24,26,28$} \\
\hline Second Opinion & no inquiry & $1,3,5-9,11-14,16,17,19,20,22,24,25,26,28$ & with inquiry & \multicolumn{3}{|l|}{$2,4,10,15,18,21,23,27$} \\
\hline Study Sign-Up & no sign-up & $24-7,9,11,13,15-17,19,21,23,24,27,28$ & with sign-up & \multicolumn{3}{|c|}{$1,3,8,10,12,14,18,20,22,25,26$} \\
\hline Record Access & no control & $1,9,13,17$ & shared control & $2,5,16,18,21,27$ & full control & $3,4,6-8,10-12,14,15,19,20,22 \cdot 26,28$ \\
\hline Records Management & no management & $1,2,4,5,7-9,13,16-19,21-28$ & with management & \multicolumn{3}{|l|}{$3,6,10-12,14,15,20$} \\
\hline Health Data Amend & review & $1,4,5,8,9,12-14,16-18,21-26,28$ & correct & $2,3,6,7,10,27$ & delete & $11,15,19,20$ \\
\hline Health Document Upload & no upload & $6,7,9,11,12,14,18-20,22,23,25-27$ & with upload & \multicolumn{3}{|c|}{$1-5,8,10,13,15-17,21,24,28$} \\
\hline
\end{tabular}

Fig. 4 Taxonomy $\mathrm{T}_{4}$ after the fourth iteration with evaluated characteristics.

\section{The TOPCOP Taxonomy}

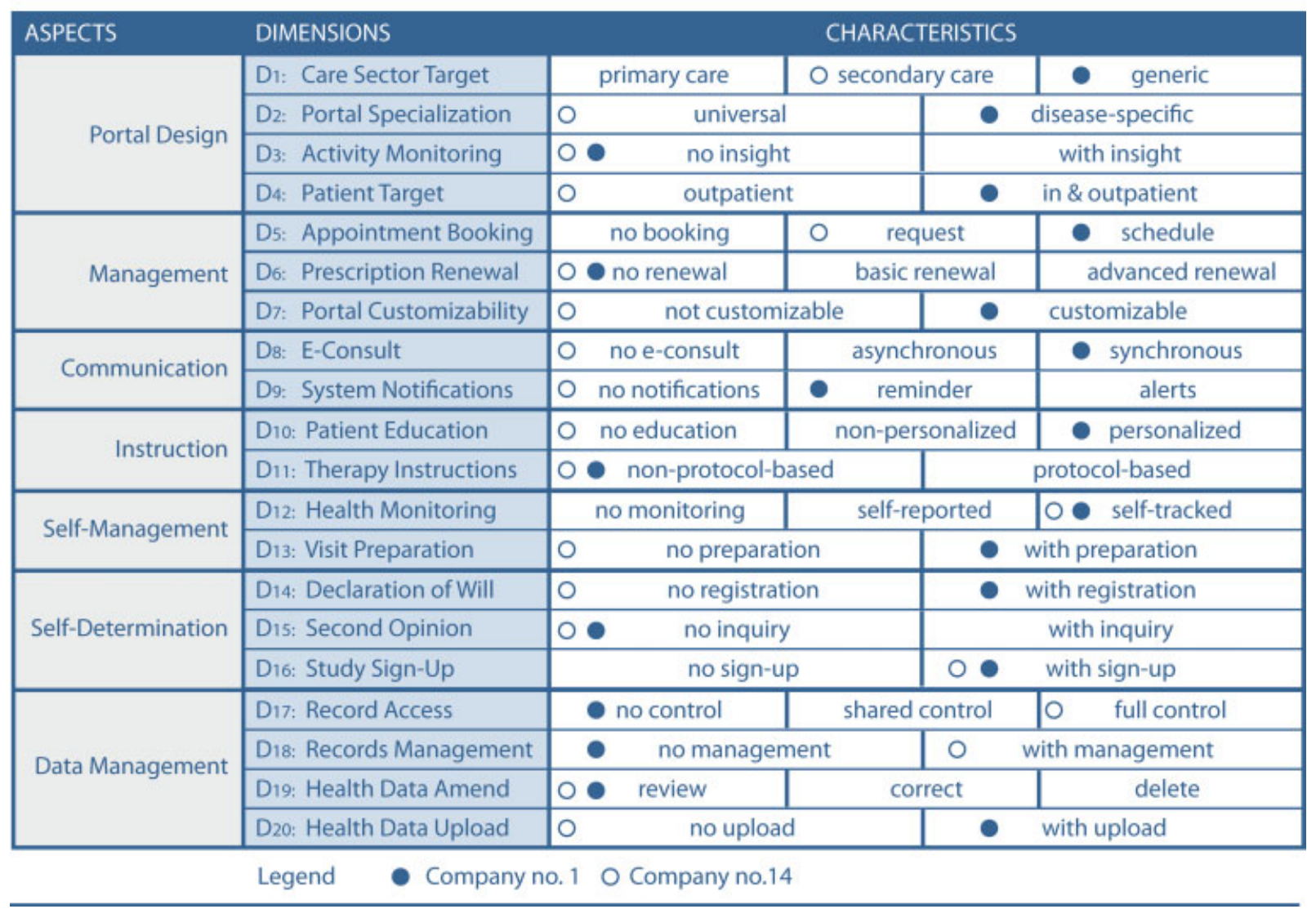

Fig. 5 Using the final TOPCOP taxonomy to compare two patient portals. TOPCOP, Taxonomy of Patient Portals based on Characteristics of Patient Engagement. 


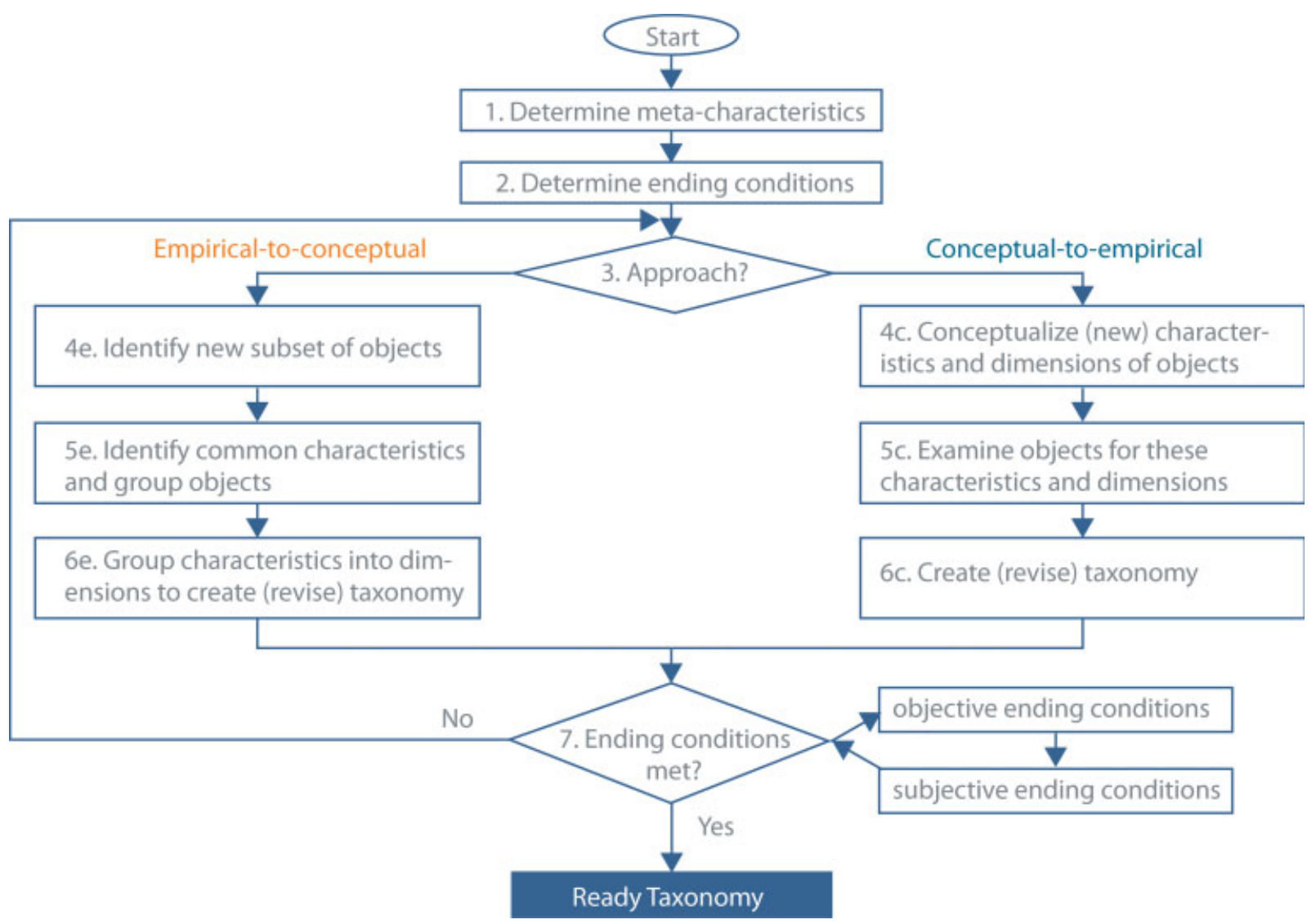

Fig. 6 The method by Nickerson et $\mathrm{al}^{23}$ of taxonomy building. Adapted with permission from Nickerson et al. ${ }^{23}$

\section{Discussion}

We developed the TOPCOP taxonomy for patient portals. This taxonomy is intended to serve health information managers to classify and compare patient portals based on characteristics appropriate to promote patient engagement. To build the taxonomy, we used a formal method that defines all steps and provides conditions for usefulness. In the first cycle, we conceptionally built a preliminary taxonomy based on a literature review. In the second cycle, we empirically evaluated our taxonomy by classifying real portals and proved the appropriateness of all characteristics. Our final taxonomy results in 20 dimensions built on 49 characteristics. Fulfilling all the ending conditions, our taxonomy is considered useful.

We identified only a few other publications related to patient portal taxonomies. All the found taxonomies were developed for different purposes than the purpose defined for this research. The purpose of using a taxonomy is the central determinant in building a taxonomy, as the purpose directly shapes the resulting taxonomy. ${ }^{23} \mathrm{~A}$ different purpose of using a taxonomy leads to a different taxonomy, and therefore the found taxonomies do not address our research aim. Thus, the TOPCOP taxonomy is the first useful taxonomy for health information managers to classify and compare patient portals focusing on characteristics promoting patient engagement. By comparing the portals, differences and similarities become apparent.

\section{Limitations}

Our approach has three limitations. The first limitation relates to the selection of software companies and health care providers to evaluate our taxonomy. Sampling was limited to companies from Germany and the United States, and to health care providers from Australia, Austria, Finland, Germany, Norway, the United Kingdom and the United States. Hence, we built our preliminary taxonomy on a broad international literature review which identified patient portals from 15 countries. The second limitation arises from the companies' low response rate when being asked to provide details on their product. We addressed this limitation by also analyzing product descriptions published online if the content was sufficiently comprehensive. However, we acknowledge that online information quality may be less precise than participating companies' information. The third limitation concerns the feature descriptions of portals available online. The available descriptions of these portals were often not quite complete, and we were unable to register with the portals as their use is restricted to register patients. However, since the intention of the fourth iteration was mainly to evaluate the appropriateness of the characteristics rather than to identify new characteristics, this limitation should not affect the outcome.

Future research should evaluate the taxonomy together with the projected users by having them apply and evaluate our taxonomy. This step is planned for the near future. Another direction for future research is to explore if the TOPCP taxonomy 
can be used by health care providers from other sectors for the same purpose. Further research may also evaluate if the taxonomy can serve as starting point to create a standard framework for patient portal functional requirement specification. Since the researchers have not found a taxonomy to categorize scientific publications related to patient portals, an additional direction for prospective research may explore whether the taxonomy can be a basis to build a taxonomy to classify and compare scientific publications. Such a taxonomy would make the published research findings more comprehensible and comparable.

Our literature review was guided by the intention to gain the broadest possible bandwidth of characteristics to build our preliminary taxonomy. We selected publications related to different types of patient portals, such as tethered portals, integrated portals of health care organizations, and national patient portals. Further, we reviewed literature referring to patient portals from 15 different countries worldwide to identify suitable characteristics. Even if the sampling for the evaluation was limited to companies and health care providers from seven countries, all conceptually derived characteristics could be confirmed. Due to the wide-ranging approach in conceptually developing our taxonomy and the empirical proof of the appropriateness of the characteristics and dimensions, we consider our taxonomy suitable to compare patient portals from any country and not only from the countries we have included in our evaluation.

\section{Conclusion}

There are still various countries, such as Germany, Italy, or Switzerland, where patient portals have not found widespread use. The landscape of available patient portals is very heterogeneous, and portals significantly vary in their general types and functionalities. We developed the TOPCOP taxonomy to help health information managers efficiently compare patient portals based on characteristics promoting patient engagement. Comparing patient portals with our taxonomy may lead to a better understanding of the differences and similarities among patient portals but may also help to recognize the various application areas and scopes of the portals. Further, the taxonomy may support health information managers to more easily define which type and functionalities of patient portal they need and guide them in their selection decision.

\section{Funding}

This research was funded in whole, or in part, by the Austrian Science Fund (FWF; no.: I 3726-N31).

\section{Conflict of Interest}

None declared.

\section{References}

1 Roehrs A, da Costa CA, Righi RD, de Oliveira KSF. Personal health records: a systematic literature review. J Med Internet Res 2017;19 (01):e13
2 Otte-Trojel T. Patient Portals Development and Outcomes in Integrated and Fragmented Health Systems. Ph.D. dissertation, Rotterdam, The Netherlands: Erasmus University Rotterdam; 2015

3 Tang PC, Ash JS, Bates DW, Overhage JM, Sands DZ. Personal health records: definitions, benefits, and strategies for overcoming barriers to adoption. J Am Med Inform Assoc 2006;13(02):121-126

4 Ammenwerth E, Hoerbst A, Lannig S, Mueller G, Siebert U, Schnell-Inderst P. Effects of adult patient portals on patient empowerment and health-related outcomes: a systematic review. Stud Health Technol Inform 2019;264(01):1106-1110

5 Rigby M, Georgiou A, Hyppönen H, et al. Patient portals as a means of information and communication technology support to patient- centric care coordination - the missing evidence and the challenges of evaluation. a joint contribution of IMIA WG EVAL and EFMI WG EVAL. Yearb Med Inform 2015;10(01): 148-159

6 Ammenwerth E, Lannig S, Hörbst A, Muller G, Schnell-Inderst P. Adult patient access to electronic health records (Protocol). Cochrane Database Syst Rev 2017;6(CD01270):1-14

7 Ammenwerth E, Schnell-Inderst P, Hoerbst A. The impact of electronic patient portals on patient care: a systematic review of controlled trials. J Med Internet Res 2012;14(06):e162

8 Alturkistani A, Greenfield G, Greaves F, Aliabadi S, Jenkins RH, Costelloe C. Patient portal functionalities and uptake: systematic review protocol. JMIR Res Protoc 2020;9(07):e14975

9 Crameri KA, Maher L, Van Dam P, Prior S. Personal electronic healthcare records: What influences consumers to engage with their clinical data online? A literature review. Health Inf Manag 2020 (e-pub ahead of print). Doi: 10.1177/1833358319895369

10 Walker DM, Sieck CJ, Menser T, Huerta TR, Scheck McAlearney A. Information technology to support patient engagement: where do we stand and where can we go? J Am Med Inform Assoc 2017; 24(06):1088-1094

11 Wass S, Vimarlund V. The role of PAEHRs in patient involvement. J Med Syst 2018;42(11):210

12 Otte-Trojel T, de Bont A, Rundall TG, van de Klundert J. What do we know about developing patient portals? a systematic literature review. J Am Med Inform Assoc 2016;23(e1):e162-e168

13 Irizarry T, DeVito Dabbs A, Curran CR. Patient portals and patient engagement: a state of the science review. J Med Internet Res 2015;17(06):e148

14 Tsai CH, Eghdam A, Davoody N, Wright G, Flowerday S, Koch S. Effects of electronic health record implementation and barriers to adoption and use: A scoping review and qualitative analysis of the content. Life (Basel) 2020;10(12):1-27

15 Wildenbos GA, Horenberg F, Jaspers M, Peute L, Sent D. How do patients value and prioritize patient portal functionalities and usage factors? A conjoint analysis study with chronically ill patients. BMC Med Inform Decis Mak 2018;18(01):108

16 Hazara AM, Durrans K, Bhandari S. The role of patient portals in enhancing self-care in patients with renal conditions. Clin Kidney J 2019;13(01):1-7

17 Fiks AG, Mayne SL, Karavite DJ, et al. Parent-reported outcomes of a shared decision-making portal in asthma: a practice-based RCT. Pediatrics 2015;135(04):e965-e973

18 Coughlin SS, Williams LB, Hatzigeorgiou C. A systematic review of studies of web portals for patients with diabetes mellitus. mHealth 2017;3(03):23-23

19 Ammenwerth E, Neyer S, Hörbst A, Mueller G, Siebert U, SchnellInderst P. Adult patient access to electronic health records. Cochrane Database Syst Rev 2021;2:CD012707

20 Scheplitz T, Benedict M, Esswein W. Patientenkompetenz durch Online-Portale - Eine Funktionsanalyse. In: Tagungsband Multikonferenz Wirtschaftsinformatik (MKWI). 2018: Data Driven X Turning Data into Value. Lüneburg: Paul Drews, Burkhardt Funk, Peter Niemeyer und Lin Xie 2018; 744-755

21 Eickhoff M, Muntermann J, Weinrich T. What do fintechs actually do? A taxonomy of fintech business models. In: Yong Jin Kim, Ritu 
Agarwal, Jae Kyu Lee. editors. Proceedings of the International Conference on Information Systems - Transforming Society with Digital Innovation, ICIS 2017, Seoul, South Korea, December 1013, 2017. Association for Information Systems, 2017

22 Glass RL, Vessey I. Contemporary application-domain taxonomies. IEEE Softw 1995;12(04):63-76

23 Nickerson RC, Varshney U, Muntermann J. A method for taxonomy development and its application in information systems. Eur J Inf Syst 2013;22(03):336-359

24 Hevner AR, March ST, Park J, Ram S. Design science in information systems research. MIS Q. 2004;28(01):75-105

25 Ammenwerth E. Die Bewertung von Informationssystemen des Gesundheitswesens: Beiträge für ein umfassendes Informations management. (Habilitation thesis), Hall, Austria: UMIT Private Universität für Medizinische Informatik und Technik Tirol; 2003

26 Bailey KD. Typologies and Taxonomies: An Introduction to Classification Techniques. Thousand Oaks, CA: Sage Publications, Inc.; 1994

27 Doty DH, Glick WH. Typologies as a unique form of theory building: toward improved understanding and modeling. Acad Manage Rev 1994;19(02):230-251

28 Lambe P. Organising Knowledge: Taxonomies, Knowledge and Organisational Effectiveness. 1st ed. Oxford, England, United Kingdom: Chandos Publishing; 2007

29 Roeder J, Muntermann J, Kneib T. Towards a taxonomy of data heterogeneity. In: 15th International Conference on Wirtschaftsinformatik. Potsdam, Germany: Gronau, N., Heine, M., Krasnova, H., Pousttchi, K.; 2020:293-308

30 Remane G, Nickerson RC, Hanelt A, Tesch JF, Kolbe LM. A Taxonomy of Carsharing Business Models. In: 2016 International Conference on Information Systems, ICIS 2016 - Digital Innovation at the Crossroads. Association for Information Systems AIS; 2016

31 Bates M, Black C, Blair F, et al. Perceptions of health information management educational and practice experiences. Perspect Health Inf Manag 2014;11(Summer):1d

32 Nickerson RC, Muntermann J, Varshney U, Issac H. Taxonomy development in information systems: developing a taxonomy of mobile applications. Accessed May 3, 2021 at: https://www.researchgate. net/publication/46479041_Taxonomy_Development_In_Information_Systems_Developing_A_Taxonomy_Of_Mobile_Applications

33 Mayring P. Qualitative Inhaltsanalyse: Grundlagen Und Techniken. 11th ed. Weinheim und Basel: Beltz Verlag; 2015

34 Mayring P. Einführung in Die Qualitative Sozialforschung. 6., Überar. Weinheim und Basel, Beltz Verlag: 2016

35 Mayring P. Qualitative Content Analysis: Theoretical Foundation, Basic Procedures and Software Solution. Accessed May 3, 2021 at: https://www.psychopen.eu/fileadmin/user_upload/books/mayring/ ssoar-2014-mayring-Qualitative_content_analysis_theoretical_foundation.pdf

36 Heithecker J. DMEA - Connecting Digital Health. Messe Berlin GmbH. https://www.dmea.de/About/DMEA/. Published 2020

37 Mold F, Raleigh M, Alharbi NS, de Lusignan S. The impact of patient online access to computerized medical records and services on type 2 diabetes: Systematic review. J Med Internet Res 2018;20(07):e235

38 Graham TAD, Ballermann M, Lang E, et al. Emergency physician use of the Alberta netcare portal, a province-wide interoperable electronic health record: Multi-method observational study.JMIR Med Inform 2018;6(03):e10184

39 Tavares J, Oliveira T. New integrated model approach to understand the factors that drive electronic health record portal adoption: cross-sectional national survey. J Med Internet Res 2018;20(11):e11032

40 Kelly MM, Dean SM, Carayon P, Wetterneck TB, Hoonakker PLT. Healthcare team perceptions of a portal for parents of hospitalized children before and after implementation. Appl Clin Inform 2017;8(01):265-278

41 Dalal AK, Dykes P, Samal L, et al. Potential of an electronic health record-integrated patient portal for improving care plan concordance during acute care. Appl Clin Inform 2019;10(03):358-366
42 Foster B, Krasowski MD. The use of an electronic health record patient portal to access diagnostic test results by emergency patients at an academic medical center: Retrospective study. J Med Internet Res 2019;21(06):e13791

43 Wallace LS, Angier H, Huguet N, et al. Patterns of electronic portal use among vulnerable patients in a nationwide practice-based research network: from the OCHIN practice-based research network (PBRN). J Am Board Fam Med 2016;29(05):592-603

44 Bell SK, Roche SD, Johansson AC, et al. Clinician perspectives on an electronic portal to improve communication with patients and families in the intensive care unit. Ann Am Thorac Soc 2016;13 (12):2197-2206

45 King G, Maxwell J, Karmali A, et al. Connecting families to their health record and care team: The use, utility, and impact of a client/family health portal at a children's rehabilitation hospital. J Med Internet Res 2017;19(04):e97

46 Miklin DJ, Vangara SS, Delamater AM, Goodman KW. Understanding of and barriers to electronic health record patient portal access in a culturally diverse pediatric population. JMIR Med Inform 2019;7(02):e11570

47 Hill JN, Smith BM, Weaver FM, et al. Potential of personal health record portals in the care of individuals with spinal cord injuries and disorders: Provider perspectives. J Spinal Cord Med 2018;41 (03):298-308

48 D'Costa SN, Kuhn IL, Fritz Z. A systematic review of patient access to medical records in the acute setting: practicalities, perspectives and ethical consequences. BMC Med Ethics 2020;21 (01):18

49 Zanaboni P, Kummervold PE, Sørensen T, Johansen MA. Patient use and experience with online access to electronic health records in Norway: results from an online survey. J Med Internet Res 2020;22(02):e16144

50 Tieu L, Schillinger D, Sarkar U, et al. Online patient websites for electronic health record access among vulnerable populations: portals to nowhere? J Am Med Inform Assoc 2017;24(e1): e47-e54

51 Chen M, Decary M. Embedding health literacy tools in patient ehr portals to facilitate productive patient engagement. Stud Health Technol Inform 2019;257:59-63

52 Leveille SG, Mejilla R, Ngo L, et al. Do patients who access clinical information on patient internet portals have more primary care visits? Med Care 2016;54(01):17-23

53 Coughlin SS, Prochaska JJ, Williams LB, et al. Patient web portals, disease management, and primary prevention. Risk Manag Healthc Policy 2017;10:33-40

54 Nambisan P. Factors that impact Patient Web Portal Readiness (PWPR) among the underserved. Int J Med Inform 2017; 102:62-70

55 Osborn CY, Mayberry LS, Mulvaney SA, Hess R. Patient web portals to improve diabetes outcomes: a systematic review. Curr Diab Rep 2010;10(06):422-435

56 Kopanitsa G, Karpov A, Lakovenko G, Laskovenko A, Yampolsky V. Exploring barriers and opportunities for adoption of web portals in Russia. Example of a tuberculosis portal. Stud Health Technol Inform 2016;224:170-174

57 Jones JB, Weiner JP, Shah NR, Stewart WF. The wired patient: patterns of electronic patient portal use among patients with cardiac disease or diabetes. J Med Internet Res 2015;17(02):e42

58 Dinh-Le C, Chuang R, Chokshi S, Mann D. Wearable health technology and electronic health record integration: scoping review and future directions. JMIR Mhealth Uhealth 2019;7 (09):e12861

59 Nøhr C, Parv L, Kink P, et al. Nationwide citizen access to their health data: analysing and comparing experiences in Denmark, Estonia and Australia. BMC Health Serv Res 2017;17(01):534

60 Gorfinkel I, Lexchin J. Enabling patient portals to access primary care medical records: Maximizing collaboration in care between patients and providers. Healthc Policy 2019;14(04):21-27 
61 Rodon Modol J. Navigating towards self-care: the Catalan public patient portal. In: Aanestad M, Grisot M, Hanseth O, Vassilakopoulou $P$, eds. Information Infrastructures within European Health Care. Switzerland: Springer; 2017:25-33

62 Grisot M, Vassilakopoulou P, Aanestad M. The Norwegian ehealth platform: development through cultivation strategies and incremental changes. In: Aanestad M, Grisot M, Hanseth O, Vassilakopoulou $P$, eds. Information Infrastructures within European Health Care. Switzerland: Springer; 2017:25-33

63 Krist AH, Peele E, Woolf SH, et al. Designing a patient-centered personal health record to promote preventive care. BMC Med Inform Decis Mak 2011;11(01):73

64 Wald JS, Businger A, Gandhi TK, et al. Implementing practicelinked pre-visit electronic journals in primary care: patient and physician use and satisfaction. J Am Med Inform Assoc 2010;17 (05):502-506

65 De Croon R, Klerkx J, Duval E. Designing a useful and usable mobile EMR application through a participatory design methodology: A case study. Proc- 2014 IEEE Int Conf Healthc Informatics, ICHI 2014;2014:176-185

66 Rodolfo I, Laranjo L, Correia N, Duarte C. Design strategy for a national integrated personal health record. In: Proceedings of the NordiCHI 2014: The 8th Nordic Conference on Human-Computer Interaction: Fun, Fast, Foundational. New York,NY, United States: Association for Computing Machinery; 2014:411-420

67 Sigler R, Kubat R, Lopez A, Newman JR. Implementation of a standardised sign-up process to increase MyChart enrolment among HIV-positive patients. BMJ Open Qual 2019;8(03): e000602

68 Sharit J, Lisigurski M, Andrade A, et al. The roles of health literacy, numeracy, and graph literacy on the usability of the VA's personal health record by veterans. J Usability Stud 2014;9:173-193

69 Kondylakis H, Flouris G, Fundulaki I, Papakonstantinou V, Tsiknakis M. Flexible access to patient data through e-consent. In: MOBIHEALTH 2015 - 5th EAI International Conference on Wireless Mobile Communication and Healthcare - Transforming Healthcare through Innovations in Mobile and Wireless Technologies. London; 2015

70 Hanauer DA, Preib R, Zheng K, Choi SW. Patient-initiated electronic health record amendment requests. J Am Med Inform Assoc 2014;21(06):992-1000

71 Miller DM, Moore SM, Fox RJ, et al. Web-based self-management for patients with multiple sclerosis: a practical, randomized trial. Telemed J E Health 2011;17(01):5-13

72 van Kuppenveld SI, van Os-Medendorp H, Tiemessen NA, van Delden JJ. Real-time access to electronic health record via a patient portal: is it harmful? A retrospective observational study. J Med Internet Res 2020;22(02):e13622

73 Nazi KM, Turvey CL, Klein DM, Hogan TP. A decade of veteran voices: Examining patient portal enhancements through the lens of user-centered design. J Med Internet Res 2018;20(07):e10413

74 Dragić L, Piljić I, Kovač M, et al. Home health smart TV - bringing ehealth closer to elders. In: 57th International Symposium ELMAR (ELMAR). Zadar; 2015:251-254

75 Ochoa A III, Kitayama K, Uijtdehaage S, et al. Patient and provider perspectives on the potential value and use of a bilingual online patient portal in a Spanish-speaking safety-net population. J Am Med Inform Assoc 2017;24(06):1160-1164

76 Bailey SC, Wallia A, Wright S, et al. Electronic health record-based strategy to promote medication adherence among patients with diabetes: longitudinal observational study. J Med Internet Res 2019;21(10):e13499

77 Senathirajah Y, Bakken S, Kaufman D. The clinician in the driver's seat: part 1 - a drag/drop user-composable electronic health record platform. J Biomed Inform 2014;52:165-176

78 Zhou L, DeAlmeida D, Parmanto B. Applying a user-centered approach to building a mobile personal health record app: development and usability study. JMIR Mhealth Uhealth 2019;7 (07):e13194

79 Ancker JS, Mauer E, Kalish RB, Vest JR, Gossey JT. Early adopters of patient-generated health data upload in an electronic patient portal. Appl Clin Inform 2019;10(02):254-260

80 O'Connell Francischetto E, Damery S, Ferguson J, Combes GmyVideoClinic randomised evaluation steering group. Video clinics versus standard face-to-face appointments for liver transplant patients in routine hospital outpatient care: study protocol for a pragmatic randomised evaluation of myVideoClinic. Trials 2018; 19(01):574

81 Bt Nasaruddin NS, Aziz IA, Rashid NA. Web-based electronic healthcare record system (EHRS) based on feedback. In: 2018 IEEE Conference on Application, Information and Network Security, AINS 2018. Langkawi, Malaysia: IEEE; 2019:27-32

82 Genes N, Violante S, Cetrangol C, Rogers L, Schadt EE, Chan YY. From smartphone to EHR: a case report on integrating patientgenerated health data. NPJ Digit Med 2018;1(01):23

83 Anthony CA, Polgreen LA, Chounramany J, et al. Outpatient blood pressure monitoring using bi-directional text messaging.J Am Soc Hypertens 2015;9(05):375-381

84 Cutrona SL, Golden JG, Goff SL, et al. Improving rates of outpatient influenza vaccination through EHR portal messages and interactive automated calls: a randomized controlled trial. J Gen Intern Med 2018;33(05):659-667

85 Wang J, Coleman DC, Kanter J, Ummer B, Siminerio L. Connecting smartphone and wearable fitness tracker data with a nationally used electronic health record system for diabetes education to facilitate behavioral goal monitoring in diabetes care: Protocol for a pragmatic multi-site randomized trial. JMIR Res Protoc 2018;7 (04):e10009

86 Khan IA. Personalized electronic health record system for monitoring patients with chronic disease. In: 2013 IEEE Systems and Information Engineering Design Symposium, SIEDS 2013. Charlottesville, VA, USA: IEEE; 2013:121-126

87 Wolff JL, Kim VS, Mintz S, Stametz R, Griffin JM. An environmental scan of shared access to patient portals. J Am Med Inform Assoc 2018;25(04):408-412

88 Brandner A, Schreiweis B, Aguduri LS, et al. The patient portal of the personal cross-enterprise electronic health record (PEHR) in the Rhine-Neckar-Region. Stud Health Technol Inform 2016; 228:157-161

89 ISO/TR 14292:2012(en) Health informatics - personal health records - definition, scope and context. Accessed May 3, 2021 at: https://www.iso.org/obp/ui/\#iso:std:iso:tr:14292:ed-1:v1:en 